\title{
Seeking informal and formal help for mental health problems in the community: a secondary analysis from a psychiatric morbidity survey in South London
}

\author{
June SL Brown ${ }^{1 *}$, Sara Evans-Lacko ${ }^{2}$, Lisa Aschan ${ }^{3}$, Max J Henderson ${ }^{3}$, Stephani L Hatch ${ }^{3}$ and Matthew Hotopf ${ }^{3}$
}

\begin{abstract}
Background: Only 30-35\% of people with mental health problems seek help from professionals. Informal help, usually from friends, family and religious leaders, is often sought but is under-researched. This study aimed to contrast patterns of informal and formal help-seeking using data from a community psychiatric morbidity survey ( $n=1692$ ) (South East London Community Health (SELCOH) Study).

Methods: Patterns of help-seeking were analysed by clinical, sociodemographic and socioeconomic indicators. Factors associated with informal and formal help-seeking were investigated using logistic regression. Cross-tabulations examined informal help-seeking patterns from different sources.

Results: 'Cases' ( $n=386)$ were participants who had scores of $\geq 12$ on the Revised Clinical Interview Schedule (CIS-R), indicating a common mental disorder. Of these, $40.1 \%$ had sought formal help, (of whom three-quarters (29\%) had also sought informal help), 33.6\% had sought informal help only and only $26.3 \%$ had sought no help. When controlling for non-clinical variables, severity, depression, suicidal ideas, functioning and longstanding illnesses were associated with formal rather than informal help-seeking. Age and ethnic group influenced sources of informal help used. Younger people most frequently sought informal help only whereas older people tended to seek help from their family. There were ethnic group differences in whether help was sought from friends, family or religious leaders.

Conclusions: This study has shown how frequently informal help is used, whether in conjunction with formal help or not. Among the 'cases', over 60\% had sought informal help, whether on its own or together with formal help. Severity was associated with formal help-seeking. Patterns of informal help use have been found. The use and effectiveness of informal help merit urgent research.
\end{abstract}

Keywords: Informal help, Formal help-seeking, Depression, Functioning, Friends, Family, Community psychiatric survey, Mental health

\section{Background}

It has been consistently found that only a third of individuals with diagnosable mental health problems seek formal help from health service providers [1-3] despite the availability of effective treatments [1]. The role of informal help from friends, families or other non-medical sources has been much less frequently researched.

\footnotetext{
* Correspondence: June.Brown@kcl.ac.uk

${ }^{1}$ Psychology Department (PO77), Kings College London, Institute of Psychiatry, De Crespigny Park, London SE5 8AF, UK

Full list of author information is available at the end of the article
}

Friends and family as well as religious leaders, or other non-health professionals usually offer informal help. It can also include self-help with other people with similar problems. Members of the public have been found to rate the helpfulness of informal help from friends and family more highly than that of professionals $([4,5])$. Informal help is more difficult to evaluate because it happens more spontaneously and therefore studies are limited. Interestingly, the World Health Organisation (WHO) assert that primary care services should be supported by 
self-care and informal community care in their optimal mix of services [6].

Kleinman [7] argues that families, friends and other community leaders as well as 'folk healers' have historically played and still play an important role in how people perceive and deal with illness or disease. The social distance between the person with the problem and the informal helper is usually less great so that there is greater agreement about the perception of the problem and how it might be handled. Similarly, Kirmayer [8] argues that how mental health services are provided to diverse groups is becoming even more important, particularly with increasing globalization. In particular, the present model is very medicalised and based on western concepts of 'illness'. Because of different uses of informal care by the different ethnic minority groups, it is argued that it will be important for services to be more 'culturally competent' [9].

There have been very few community surveys examining the use of formal and informal help, that is help from family, friends and spiritual or religious leaders. There have been a large number of studies on informal help but they have focused on specific disadvantaged groups, such as gay men with HIV [10] or partner abuse [11] or demographic groups such as young people [12], and ethnic minority groups $[13,14]$. Seeking help from multiple sources has also been found [13].

In the only community study in the UK to date investigating informal help among adults, Oliver et al. [15] found that $63.1 \%$ of 10302 participants preferred to seek help from friends and family when they were feeling 'stress and strain'. Using the General Health Questionnaire (GHQ) [16] to measure severity, they found no differences in problem severity amongst those seeking informal help, but found differences with formal help, with $14 \%$ with less severe problems having sought formal help compared to $28 \%$ with more severe problems. In a smaller study using a psychiatric interview to assess severity, Rudell and colleagues [14] also found that informal help was commonly used, with talking to friends and family and keeping busy the most common strategies used.

Relatively little is known about its determinants and its effectiveness. Further, it is not clear where informal help fits into the current system of care. It may be that it is used as a precursor to formal help, or alternatively, it may be used alongside formal help. On the other hand, there is also some evidence that informal help prevents access to formal help, such that evidence-based treatments are not utilized. Lamb et al. [17] found that the low access to formal help of 'hard to reach' groups such as black and minority ethnic groups and depressed elderly people, was partly explained by these groups perceiving their problems as rooted in social problems and attempting to manage their problems themselves. They often sought help from close family and became isolated from other networks, rather than seeking formal help.

The characteristics of individuals who seek formal help are better understood. While severity of mental health problems is the most consistent predictor of formal help-seeking [18] other clinical variables have also been found to be relevant including functioning [18], perceived need [19] as well as co-morbidity [2]. Sociodemographic characteristics are also associated with help-seeking, with men more reluctant than women to seek formal help [18]. Ethnic differences have also been found with Asians tending to present less frequently in primary care settings even when controlling for severity [18] and GPs being less good at detecting the mental health problems of black Caribbean people [20]. People with diagnoses of depression have been found to be most likely to seek formal help compared with other mental disorders [18].

The aim of this study therefore is to investigate factors associated with informal help-seeking for mental health problems and contrast these to correlates of formal help-seeking using data from a community survey. The factors were socio-demographic, economic and clinical indicators. We also sought to explore the type of informal help people used.

\section{Method \\ Design}

We analysed data from a cross-sectional study of mental and physical health: the South East London Community Health (SELCoH) Study.

\section{Hypotheses}

We set out to test the following hypotheses:

1. Compared to those who use formal help, exclusive use of informal help would be associated with less severe mental disorder.

2. Compared to those who use formal help, exclusive use of informal help would be associated with higher social support.

3. There would be sociodemographic factors (age, gender and ethnic group) with younger, female and black and ethnic minority groups being more likely to seek informal help.

4. There would be socioeconomic differences in helpseeking patterns with lower SES groups (characterised by low income and no qualifications) being more likely to seek formal help compared to informal help only.

\section{Setting and study participants}

The South East London Community Health (SELCoH) study is a community survey of psychiatric and physical morbidity of 1698 adults, aged 16 years and over from 
1075 randomly selected households in South London boroughs of Southwark and Lambeth. Data were collected between 2008-2010, applying similar methods to the British National Psychiatric Morbidity Surveys [21]; study methods are described in detail elsewhere [22,23].

In the two boroughs, there is higher deprivation than the England average, but similar proportions of economically active and inactive residents to greater London $[24,25]$. The boroughs are ethnically diverse, with a greater number of Black Caribbean and Black African residents but fewer South Asian residents than other areas of London [26,27]. The achieved SELCoH study sample was representative of the catchment area with regard to 2011 UK census demographic and socioeconomic indicators, with the exception of the study sample being slightly younger and including more students among the economically inactive (42.0\% vs $33.3 \%$ ).

Ethical approval was not sought for this study because we were performing a secondary analysis of data that had already been collected. The original study had received approval from the King's College London research ethics committee, reference CREC/07/08-152.

\section{Measures \\ Dependent variables: use of formal and/or informal help in past year}

Help-seeking within the past year was determined by self-report. 'Formal help-seeking' was tapped by a question: "In the past 12 months, have you spoken to a GP or family doctor, a psychological therapist/counsellor or other sources of help on your own behalf, either in person or by telephone about being anxious or depressed or a mental, nervous or emotional problem?"

Informal help-seeking was gauged by responses to: "In the past 12 months have you gone and seen any of the following for an emotional problem? Options included friends, family members, or spiritual/religious leaders".

Because of the overlap of informal and formal helpseeking, we used four mutually exclusive help-seeking categories - no help, informal help only, both informal and formal help, or formal help only. In the regression analysis, the no help group was excluded and the three help-seeking groups were collapsed into informal help only and contrasted with formal help (with and without informal help).

\section{Potential predictors of help-seeking}

Clinical and non-clinical characteristics were investigated. Clinical variables examined included psychiatric severity, psychiatric diagnoses, suicidal indicators, longstanding illness and functioning indicators. 'Non-clinical' variables included sociodemographic characteristics, socioeconomic characteristics, and social support.

\section{Clinical variables}

Psychiatric symptoms and diagnosis

Revised Clinical Interview Schedule (CIS-R) The CIS-R [28] is a structured interview assessing psychiatric symptom status during the past month, and was used to assess severity of mental disorder. A total CIS-R score of 12 or above is conventionally used to indicate the presence of common mental disorder (CMD) (to be referred to as 'cases' for subsample analyses). We further categorized individuals scoring above the threshold into having severe CMD (18+), mild/moderate CMD (12-17) or being healthy (0-11) and used this measure as an independent variable.

The CIS-R provides ICD-10 diagnoses for ten psychiatric disorders through a standard algorithm. However, because of the very small numbers of people experiencing some disorders, only the four most common diagnoses were used for this study: depression (11.9\%), non-specified neurotic disorder $(6.63 \%)$, generalized anxiety disorder (3.51\%) and phobia (1.73\%).

\section{Physical health and functioning}

The global health item on the Short Form Health Survey SF-12 [29] was used to assess global health status. This item asked participants to rate their health on a five point scale from 'poor' to 'excellent'. The variable was categorised as either Fair/poor vs Good/very good/excellent. We used two disability measures: functional limitations due to emotional health measured on the SF-12, and problems with activities in daily living (ADL) indicating limitations in five domains (personal help, transport, medical help, household activities and money). For these analyses, a cut-off of three ADL problems or more was used to indicate problems [18].

\section{Other clinical indicators \\ Suicidal ideation}

Past-year suicidal ideation was measured through a single item question, replicating the measure from the Adult Psychiatric Morbidity Survey [21].

\section{Hazardous alcohol use}

This was assessed through the Alcohol use Disorders Identification Test (AUDIT), a 10 item measure of alcohol consumption, dependence and misuse over the past year, with scores ranging from 0-40. Hazardous alcohol use was defined by scores of 8 or more [30].

\section{Past-year drug use}

This was indicated through self-reported use of any of the following illicit drugs in the past year: cannabis, amphetamines, cocaine, ecstasy, LSD, tranquillizers, crack, and heroin. 


\section{Long-standing illness}

Participants were asked to report if they had any longstanding illness, disability or infirmity that had troubled or was likely to affect the participant over a period of time. The list included high blood pressure, bronchitis, heart trouble, cancer etc.

\section{Non-clinical variables}

Sociodemographic variables included age, gender, ethnicity, relationship status, and migrant status.

Age was measured continuously and also categorized into 5 groups. Ethnicity was categorised into 5 groups. Current relationship status was categorized as married/ cohabiting vs. not. Migrant status indicated whether or not the person was born in the UK.

Socioeconomic indicators included education, employment status and household income. There were 4 employment categories and 4 educational categories. Income was measured as the gross annual household income from all sources before any deductions, and categorized into 5 categories.

Social support Presence of emotional social support was indicated by 2 items: having someone to talk to about something that was bothering you or when you felt lonely and wanted some company; and having someone who makes you feel good, loved or cared for.

\section{Analysis}

All analyses were carried out in Stata 11 and accounted for household clustering and non-response using survey weights and applying 'svy' commands in order to generate robust standard errors [31]. The prevalence of informal and formal and sources of informal help were estimated within the full sample and within 'cases'. The prevalence of informal sources of help was also estimated within the subsample of informal help users.

In generating the four mutually exclusive utilisation categories (no help, informal help only, both informal and formal, only formal), the sample size was reduced from 1698 to 1610 due to missing observations (86 true missing from the informal help item; two refusals to answer the formal help question). Within this sample, percentage prevalence estimates of informal and formal help use were described by sociodemographic, socioeconomic and clinical variables using Pearson's $x^{2}$ test with Rao \& Scott corrections to test for differences.

Unadjusted and fully adjusted logistic regression analyses were carried out in order to estimate factors associated with using informal help only versus any formal help (including combined informal and formal help). Adjusted estimations of socio-demographic and SES associations controlled for each other without adjusting for health related indicators or social support. Where clinical/health related and social support indicators were the independent variables of interest, they were entered separately from each other, only controlling for sociodemographics and SES to avoid collinearity. These models adjusted for age using the continuous measure, rather the categorical measure used to adjust for sociodemographic and socio-economic associations. All of the adjusted models were tested for goodness-of-fit using the post-hoc 'svylogitgof' command, which is appropriate for survey data [32].

Finally, we described use of specific sources of informal help (i.e., family, friend, religious leader, other) by gender, age, ethnicity and migrant status by calculating percentage prevalence estimates.

\section{Results}

Informal and formal help-seeking patterns Participant characteristics

Table 1 shows the prevalence of the different types of help seeking.

For the sample as a whole, informal help was sought twice as frequently (36.1\%) as formal help (17.5\%). Of those who sought formal help, the majority used informal help as well (69.3\%), whereas most people who sought informal help did not use formal help (65.1\%).

Among 'cases' $(n=386), 33.6 \%$ had sought informal help only. Of the $40.1 \%$ of 'cases' who had sought formal help, three-quarters (29\%) had also sought informal help, meaning only $11.1 \%$ sought formal help alone. Only $26.3 \%$ had sought no help. The most frequent form of informal help used was from friends or family with a small minority consulting religious leaders.

Sociodemographic and socio-economic patterns for the 4 help-seeking groups are described in Table 2. Men were less likely to seek help than women, the differences being particularly pronounced with informal help. Younger people more frequently sought informal help only. The 56 and older group sought no help most frequently, and they used a slightly higher proportion of formal help to informal help. The 26-40 (19.7\%) and 41-55 (22.2\%) year old age groups sought formal help most frequently, whether in combination with informal help or not.

There were no significant differences by migrant status or ethnicity. Relationship status differentiated the groups. Non-married or non-cohabiting participants were more likely to seek formal and/or informal help (20.7\%) or informal help only (25.3\%).

In terms of socio-economic differences, the unemployed group was much more likely to seek formal - as well as informal - help (total 54.5\%) than the other employment groups whereas employed participants tended to seek informal help only $(24.5 \%)$ or no help $(60.4 \%)$. Marginally significant differences across education qualification levels were found. Those with no qualifications tended to be less likely to seek any form of help, particularly informal help 
Table 1 Prevalence of help seeking

\begin{tabular}{|c|c|c|c|c|c|c|c|c|c|}
\hline & \multicolumn{3}{|c|}{ Full sample $(\mathrm{N}=1698)$} & \multicolumn{3}{|c|}{ CIS-R $\geq 12(n=396)$} & \multicolumn{3}{|c|}{ Informal help users $(n=577)$} \\
\hline & $n$ & $\%$ & $(95 \% \mathrm{Cl})$ & $n$ & $\%$ & $(95 \% \mathrm{Cl})$ & $n$ & $\%$ & $(95 \% \mathrm{Cl})$ \\
\hline \multicolumn{10}{|l|}{ Type of help* } \\
\hline Overall formal help & 290 & 17.5 & $(15.7-19.5)$ & 156 & 39.4 & $(34.4-44.5)$ & & - & \\
\hline Overall informal help & 579 & 36.1 & $(33.5-38.7)$ & 239 & 62.5 & $(57.2-67.5)$ & & - & \\
\hline \multicolumn{10}{|l|}{ Help composite variable } \\
\hline Formal help only & 88 & 5.6 & $(4.5-7.0)$ & 42 & 11.1 & $(8.2-15.0)$ & & - & \\
\hline Informal help and formal help & 201 & 12.8 & $(11.2-14.6)$ & 113 & 29.0 & $(24.6-33.7)$ & & - & \\
\hline Informal help only & 377 & 23.3 & $(21.1-25.6)$ & 126 & 33.6 & $(28.8-38.8)$ & & - & \\
\hline No help & 944 & 58.3 & $(55.7-61.0)$ & 105 & 26.3 & $(21.9-31.4)$ & & - & \\
\hline \multicolumn{10}{|l|}{ Informal help use } \\
\hline Friend & 397 & 24.1 & $(21.9-26.5)$ & 160 & 40.1 & $(35.1-45.4)$ & 397 & 67.0 & $(62.7-71.1)$ \\
\hline Family & 370 & 23.4 & $(21.2-25.7)$ & 158 & 42.1 & $(36.8-47.5)$ & 370 & 64.9 & $(60.7-68.9)$ \\
\hline Religious leaders & 27 & 1.9 & $(1.2-2.8)$ & 13 & 3.3 & $(1.8-5.8)$ & 27 & 5.2 & $(3.5-7.8)$ \\
\hline Other & 42 & 2.4 & $(1.8-3.3)$ & 14 & 3.1 & $(1.8-5.3)$ & 42 & 6.6 & $(4.9-9.0)$ \\
\hline
\end{tabular}

Frequencies show actual counts; percentages have been weighted.

p-values show significance level of Pearson's Chi square test with Rao \& Scott corrections.

*with overlaps of formal and informal help.

on its own. Income differences were significant, with the lowest income group being most likely to seek formal help (with or without informal help) but made less use of informal help on its own than other groups.

Clinical differences across the 4 help-seeking groups are shown in Table 3. Participants scoring above the threshold on the CIS-R (those categorized as 12-17 or $18+$ ) were more likely than those below the threshold to seek formal help, whether on its own or with informal help. Compared to those with scores below 12, they were more likely to seek informal help only and less likely to seek no help. While those with CIS-R scores below 12 were proportionately least likely to seek any help, 45 (3.8\%) had sought formal help. Looking at it another way, of the 88 individuals who had sought formal help only, 45 (51.1\%) scored below the threshold. Similarly, of the 201 who had sought both informal and formal help, 88 (43.8\%) were below the threshold.

Individuals with diagnoses of depression and with suicidal ideation tended to be more likely to use formal help, with about half seeking formal help. Those with suicidal ideation were also significantly less likely to seek informal help only. Participants with long-standing illnesses tended to seek formal help, whether with or without informal help more often, and informal help alone less often, compared to those without these problems. Those reporting functional limitations due to emotional health and activities in daily living also indicated increased use of all types of help (informal help only, both informal and formal help, and formal help only). We found that those with someone to talk to tended to seek informal help rather than formal help. Conversely, those who did not have someone to talk to, tended to seek formal help. However, no differences were found between those who did and did not endorse the item about whether they had someone to make them feel cared for.

Table 4 describes results of the logistic regression and factors associated with exclusive informal help seeking versus those who sought formal help and gives the unadjusted and adjusted results. All adjusted models had acceptable goodness-of-fit ( $p>0.05$ ). The degrees of freedom for these tests were 9 and within the range of 465-8. The adjusted results show that those with CIS-R scores above the threshold, any primary diagnosis, a depression diagnosis, suicidal ideation, longstanding illnesses, functional limitations and poor perceived health were less likely to seek informal help, but seek formal help. Contrary to prediction, social support was not associated with exclusive informal help seeking.

Table 5 explores socio-demographic variables by source of informal help. When the pattern of informal help was examined, regardless of caseness, significant age differences were found. The 16-25 year olds were much more likely to seek help from friends $(81.9 \%)$ whereas family members were used mainly by older people (70.5\%). Religious leaders were most often used by those aged 41 and above (16.6\%). Different patterns of informal and formal help-seeking were also shown according to ethnic groups. Black Caribbeans tended to seek help from friends (77.9\%) and were less likely to use family members. In contrast, Asians tended to use family members (88.4\%) but not friends. Religious leaders were most likely to be used by black Africans (17.8\%), Asians (14.9\%) and by migrants $(8.7 \%)$. 
Table 2 The socio-demographic and socio-economic distribution of formal and informal help seeking $(N=1,610)$

\begin{tabular}{|c|c|c|c|c|c|c|c|c|c|c|c|c|c|c|c|}
\hline & \multirow[b]{2}{*}{$\mathbf{N}$} & \multicolumn{3}{|c|}{$\begin{array}{l}\text { Formal only } \\
(n=88)\end{array}$} & \multicolumn{3}{|c|}{$\begin{array}{l}\text { Formal and informal } \\
(\mathrm{n}=377)\end{array}$} & \multicolumn{3}{|c|}{$\begin{array}{l}\text { Informal only } \\
(n=201)\end{array}$} & \multicolumn{3}{|c|}{ No help $(n=944)$} & \multirow[b]{2}{*}{$x^{2}$} & \multirow[b]{2}{*}{$p$} \\
\hline & & $n$ & $\%$ & $(95 \% \mathrm{Cl})$ & $n$ & $\%$ & $(95 \% \mathrm{Cl})$ & $\mathbf{n}$ & $\%$ & $(95 \% \mathrm{Cl})$ & $\mathbf{n}$ & $\%$ & $(95 \% \mathrm{Cl})$ & & \\
\hline \multicolumn{16}{|l|}{ Socio-demographic } \\
\hline Gender & & & & & & & & & & & & & & 11.63 & $<0.001$ \\
\hline Male & 702 & 36 & 5.2 & $(3.7-7.2)$ & 65 & 8.9 & $(7.0-11.3)$ & 130 & 17.9 & $(15.3-20.9$ & 471 & 67.9 & $(64.3-71.3)$ & & \\
\hline Female & 908 & 52 & 5.8 & $(4.4-7.6)$ & 136 & 14.7 & $(12.5-17.2)$ & 247 & 25.9 & $(23.0-29.0)$ & 473 & 53.6 & $(50.1-57.0)$ & & \\
\hline Age & & & & & & & & & & & & & & 4.09 & $<0.001$ \\
\hline $16-25$ & 387 & 16 & 4.2 & $(2.6-6.7)$ & 43 & 11.8 & $(8.7-15.8)$ & 108 & 28.8 & $(24.3-33.6)$ & 220 & 55.2 & $(50.1-60.2)$ & & \\
\hline $26-40$ & 521 & 24 & 4.6 & $(3.1-6.9)$ & 76 & 15.1 & $(12.2-18.7)$ & 141 & 28.1 & $(24.2-32.4)$ & 280 & 52.1 & $(47.5-56.6)$ & & \\
\hline $41-55$ & 398 & 29 & 7.4 & $(5.1-10.4)$ & 55 & 14.8 & $(11.5-18.9)$ & 80 & 20.7 & $(16.9-25.1)$ & 234 & 57.1 & $(52.0-62.1)$ & & \\
\hline 56 or older & 304 & 19 & 6.2 & $(3.9-9.6)$ & 27 & 9.4 & $(6.5-13.5)$ & 48 & 16.5 & $(12.5-21.3)$ & 210 & 68.0 & $(62.1-73.3)$ & & \\
\hline Ethnic group & & & & & & & & & & & & & & 1.10 & 0.356 \\
\hline White & 987 & 58 & 6.1 & $(4.7-7.9)$ & 133 & 13.5 & $(11.3-15.9)$ & 231 & 23.2 & $(20.5-26.2)$ & 565 & 57.2 & $(53.8-60.6)$ & & \\
\hline Black Caribbean & 137 & 11 & 8.0 & $(4.4-14.2)$ & 14 & 11.1 & $(6.7-17.8)$ & 34 & 24.1 & $(17.0-32.9)$ & 78 & 56.8 & $(47.8-65.5)$ & & \\
\hline Black African & 225 & 11 & 4.7 & $(2.6-8.4)$ & 21 & 10.3 & $(6.7-15.6)$ & 46 & 19.2 & $(14.5-25.0)$ & 147 & 65.7 & $(58.6-72.2)$ & & \\
\hline Asian & 60 & 0 & - & & 7 & 12.9 & $(6.3-24.5)$ & 20 & 33.7 & $(21.9-48.0)$ & 33 & 53.4 & $(40.1-66.3)$ & & \\
\hline Other & 199 & 8 & 4.0 & $(2.0-7.9)$ & 25 & 12.8 & $(8.8-18.3)$ & 45 & 23.8 & $(17.9-30.9)$ & 121 & 59.4 & $(52.1-66.4)$ & & \\
\hline Relationship status & & & & & & & & & & & & & & 4.48 & 0.004 \\
\hline Married/cohabitating & 739 & 32 & 4.7 & $(3.3-6.7)$ & 79 & 11.0 & $(8.8-13.6)$ & 154 & 20.8 & $(17.9-24.0)$ & 474 & 63.5 & $(59.6-67.2)$ & & \\
\hline Non-married/non-cohabitating* & 871 & 56 & 6.4 & $(4.9-8.3)$ & 122 & 14.3 & $(12.0-17.0)$ & 233 & 25.3 & $(22.3-28.6)$ & 470 & 54.0 & $(50.3-57.6)$ & & \\
\hline Migration status & & & & & & & & & & & & & & 2.07 & 0.102 \\
\hline Non-migrant & 970 & 56 & 6.0 & $(4.6-7.8)$ & 120 & 12.4 & $(10.4-14.8)$ & 206 & 21.4 & $(18.8-24.3)$ & 588 & 60.2 & $(56.8-63.5)$ & & \\
\hline Migrant & 633 & 29 & 4.6 & $(3.2-6.6)$ & 81 & 13.5 & $(10.9-16.6)$ & 170 & 26.2 & $(22.8-30.0)$ & 353 & 55.7 & $(51.4-59.9)$ & & \\
\hline \multicolumn{16}{|l|}{ Socio-economic } \\
\hline Employment status & & & & & & & & & & & & & & 3.03 & 0.001 \\
\hline Employed & 863 & 38 & 4.6 & $(3.3-6.2)$ & 87 & 10.5 & $(8.5-12.8)$ & 210 & 24.5 & $(21.6-27.7)$ & 528 & 60.4 & $(56.9-63.9)$ & & \\
\hline Unemployed & 164 & 16 & 10.2 & $(6.2-16.2)$ & 31 & 18.9 & $(13.5-25.7)$ & 41 & 25.4 & $(19.0-33.1)$ & 76 & 45.6 & $(37.8-53.5)$ & & \\
\hline Student & 239 & 12 & 4.9 & $(2.7-8.7)$ & 28 & 12.7 & $(8.8-18.0)$ & 63 & 26.9 & $(21.5-33.1)$ & 136 & 55.5 & $(48.9-61.9)$ & & \\
\hline Other & 336 & 22 & 6.4 & $(4.2-9.8)$ & 53 & 14.8 & $(11.3-19.1)$ & 61 & 18.2 & $(14.2-23.0)$ & 200 & 60.6 & (54.9-65.9) & & \\
\hline Education & & & & & & & & & & & & & & 1.90 & 0.049 \\
\hline No qualifications & 230 & 16 & 6.5 & $(3.9-10.7)$ & 28 & 12.0 & $(8.2-17.2)$ & 37 & 15.9 & $(11.5-21.6)$ & 149 & 65.6 & (58.8-71.8) & & \\
\hline GCSE & 318 & 24 & 7.9 & $(5.2-11.7)$ & 43 & 13.7 & $(10.2-18.2)$ & 78 & 24.8 & $(20.2-30.0)$ & 173 & 53.6 & $(47.9-59.3)$ & & \\
\hline A-level & 407 & 23 & 5.7 & $(3.8-8.6)$ & 53 & 13.5 & $(10.2-17.6)$ & 100 & 25.1 & $(20.9-29.8)$ & 231 & 55.7 & $(50.6-60.7)$ & & \\
\hline Degree or above & 655 & 25 & 4.0 & $(2.7-5.9)$ & 77 & 12.2 & $(9.8-15.1)$ & 162 & 24.7 & $(21.5-28.3)$ & 391 & 59.1 & $(55.1-63.0)$ & & \\
\hline Annual household income & & & & & & & & & & & & & & 2.38 & 0.005 \\
\hline$£ 0-5,475$ & 133 & 11 & 7.1 & $(3.9-12.6)$ & 36 & 27.3 & $(19.8-36.3)$ & 20 & 16.1 & $(10.3-24.4)$ & 66 & 49.5 & $(40.4-58.6)$ & & \\
\hline$£ 5,476-12,097$ & 201 & 14 & 6.6 & $(3.8-11.2)$ & 31 & 15.1 & $(10.7-21.1)$ & 48 & 23.2 & $(17.7-29.9)$ & 108 & 55.0 & $(47.7-62.1)$ & & \\
\hline$£ 12,098-20,753$ & 196 & 7 & 4.0 & $(1.9-8.4)$ & 23 & 12.2 & $(8.1-17.9)$ & 49 & 24.5 & $(18.8-31.4)$ & 117 & 59.3 & $(51.8-66.3)$ & & \\
\hline$£ 20,754-31,494$ & 167 & 11 & 6.4 & $(3.5-11.3)$ & 16 & 9.9 & $(6.1-15.7)$ & 43 & 25.2 & $(18.9-32.8)$ & 97 & 58.5 & $(50.4-66.2)$ & & \\
\hline$£ 31,495$ or more & 662 & 30 & 4.7 & $(3.3-6.7)$ & 75 & 11.6 & $(9.3-14.4)$ & 156 & 23.6 & $(20.4-27.1)$ & 401 & 60.1 & $(56.2-63.9)$ & & \\
\hline
\end{tabular}

Frequencies show actual counts; percentages have been weighted.

Counts may not add up due to missing values.

$X^{2}$ statistics and $p$-values are weighted outcomes from Pearson's Chi square tests with Rao \& Scott corrections.

*Non-married/cohabitating category include single, divorced/separated, and widowed relationship status. 
Table 3 The distribution of formal and informal help seeking by health and social support indicators $(N=1,610)$

\begin{tabular}{|c|c|c|c|c|c|c|c|c|c|c|c|c|c|c|}
\hline \multirow[b]{2}{*}{$N$} & \multicolumn{3}{|c|}{$\begin{array}{l}\text { Formal only } \\
(n=88)\end{array}$} & \multicolumn{3}{|c|}{$\begin{array}{l}\text { Formal and informal } \\
(n=201)\end{array}$} & \multicolumn{3}{|c|}{$\begin{array}{l}\text { Informal only } \\
(n=377)\end{array}$} & \multicolumn{3}{|c|}{ No help $(n=944)$} & \multirow[b]{2}{*}{$x^{2}$} & \multirow[b]{2}{*}{$p$} \\
\hline & $\mathrm{n}$ & $\%$ & $(95 \% \mathrm{Cl})$ & $\mathrm{n}$ & $\%$ & $(95 \% \mathrm{Cl})$ & $\mathrm{n}$ & $\%$ & $(95 \% \mathrm{Cl})$ & $\mathrm{n}$ & $\%$ & $(95 \% \mathrm{Cl})$ & & \\
\hline
\end{tabular}

\section{Clinical indicators}

\section{CMD (CIS-R score)}

No CMD $(<12)$

CMD (12-17)

Symptoms likely to require treatment $(\geq 18)$

Any CIS-R primary diagnosis

No

Yes

Non-specified neurotic disorder

No

Yes

Generalised anxiety disorder

No

Yes

Phobia (any)

No

Yes

\section{Depression}

No

Yes

Other $^{\dagger}$

No

Yes

Suicidal ideation

No

Yes

Hazardous alcohol use

No

Yes

Drug use (past year)

No

Yes

Long-standing illness

No

Yes

\section{Self-rated health}

Good or better

Fair or poor

ADL problems with:

Personal help

No

Yes
$40.20<0.001$

$\begin{array}{lllllllllllll}1,219 & 45 & 3.8 & (2.8-5.1) & 88 & 7.5 & (6.0-9.2) & 250 & 19.9 & (17.6-22.3) & 836 & 68.9 & (66.0-71.6) \\ 188 & 17 & 9.5 & (5.8-15.0) & 39 & 21.8 & (16.3-28.5) & 66 & 35.9 & (29.0-43.5) & 66 & 32.9 & (26.1-40.5) \\ 198 & 25 & 12.6 & (8.5-18.2) & 74 & 35.5 & (29.1-42.6) & 60 & 31.4 & (25.1-38.5) & 39 & 20.4 & (15.1-27.0)\end{array}$

$74.81<0.001$

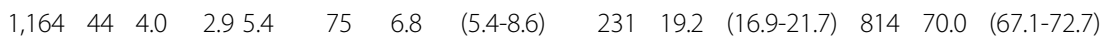

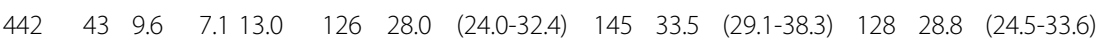

$5.11 \quad 0.002$

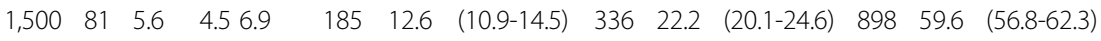

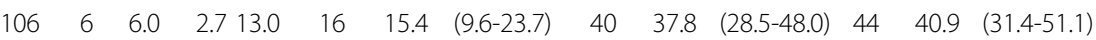

$8.56<0.001$

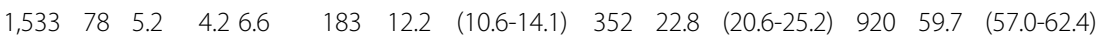

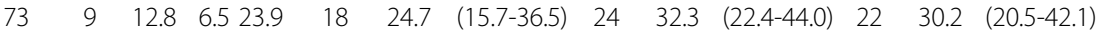

$2.83 \quad 0.037$

$\begin{array}{lllllllllllll}1,559 & 86 & 5.7 & 4.67 .1 & 190 & 12.5 & (10.9-14.4) & 359 & 23.0 & (20.8-25.3) & 924 & 58.8 & (56.1-61.5)\end{array}$

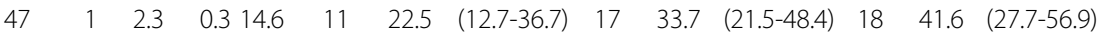

$52.28<0.001$

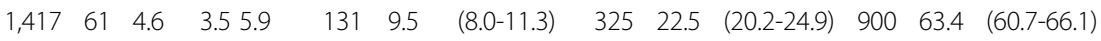

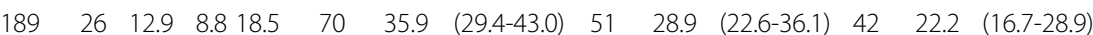

$12.40<0.001$

$\begin{array}{lllllllllllll}1,579 & 86 & 5.6 & 4.57 .0 & 190 & 12.4 & (10.7-14.2) & 363 & 22.7 & (20.6-25.0) & 940 & 59.3 & (56.6-61.9)\end{array}$

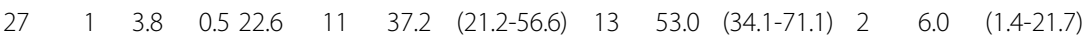

$21.53<0.001$

$\begin{array}{lllllllllllll}1,518 & 77 & 5.2 & (4.2-6.6) & 164 & 11.2 & (9.6-13.1) & 363 & 23.8 & (21.5-26.2) & 914 & 59.7 & (57.0-62.5)\end{array}$

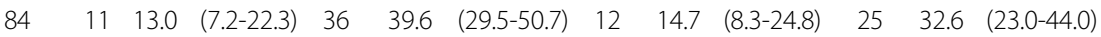

$4.50 \quad 0.002$

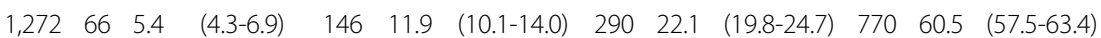

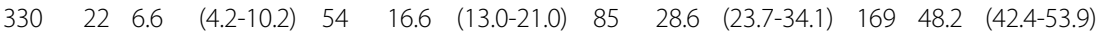

$8.96<0.001$

$\begin{array}{lllllllllllll}1,261 & 66 & 5.5 & (4.3-7.0) & 142 & 11.7 & (9.9-13.7) & 273 & 21.5 & (19.2-24.1) & 780 & 61.3 & (58.4-64.2)\end{array}$

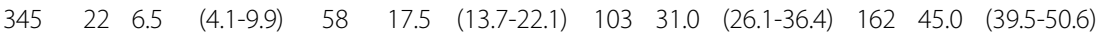

$10.12<0.001$

$\begin{array}{lllllllllllll}959 & 42 & 4.8 & (3.5-6.5) & 82 & 8.6 & (7.0-10.6) & 238 & 24.8 & (22.0-27.8) & 597 & 61.7 & (58.4-64.9)\end{array}$

$\begin{array}{lllllllllllll}643 & 46 & 6.7 & (4.9-8.9) & 117 & 17.6 & (14.7-20.9) & 137 & 21.4 & (18.2-24.9) & 343 & 54.3 & (50.1-58.5)\end{array}$

$20.22<0.001$

$\begin{array}{lllllllllllll}1,314 & 59 & 4.7 & (3.6-6.0) & 131 & 10.0 & (8.4-11.8) & 303 & 22.7 & (20.4-25.2) & 821 & 62.7 & (59.8-65.5)\end{array}$

$\begin{array}{lllllllllllll}288 & 28 & 9.4 & (6.5-13.4) & 68 & 23.7 & (19.0-29.1) & 73 & 25.8 & (20.9-31.5) & 119 & 41.1 & (35.2-47.3)\end{array}$

3.690 .012

$\begin{array}{lllllllllllll}1,531 & 84 & 5.7 & (4.6-7.1) & 183 & 12.1 & (10.4-13.9) & 359 & 23.3 & (21.1-25.7) & 905 & 58.9 & (56.2-61.6)\end{array}$

$\begin{array}{lllllllllllll}61 & 3 & 4.2 & (1.3-12.3) & 16 & 26.4 & (16.5-39.5) & 14 & 24.0 & (14.5-37.1) & 28 & 45.4 & (32.7-58.7)\end{array}$ 
Table 3 The distribution of formal and informal help seeking by health and social support indicators $(N=1,610)$ (Continued)

\begin{tabular}{|c|c|c|c|c|c|c|c|c|c|c|c|c|c|c|c|}
\hline Using transport & & & & & & & & & & & & & & 6.60 & $<0.001$ \\
\hline No & 1,508 & 76 & 5.1 & $(4.0-6.4)$ & 179 & 12.1 & $(10.4-13.9)$ & 353 & 23.2 & $(21.0-25.6)$ & 900 & 59.6 & $(56.8-62.3)$ & & \\
\hline Yes & 84 & 11 & 13.0 & $(7.1-22.4)$ & 20 & 22.8 & $(14.8-33.6)$ & 20 & 24.8 & $(16.4-35.6)$ & 33 & 39.4 & $(29.2-50.7)$ & & \\
\hline Medical help & & & & & & & & & & & & & & 1.86 & 0.134 \\
\hline No & 1,567 & 87 & 5.7 & $(4.6-7.1)$ & 193 & 12.5 & $(10.9-14.4)$ & 365 & 23.1 & $(21.0-25.5)$ & 922 & 58.6 & $(55.9-61.3)$ & & \\
\hline Yes & 25 & 0 & - & & 6 & 24.7 & $(11.0-46.5)$ & 8 & 31.9 & $(16.1-53.3)$ & 11 & 43.4 & $(24.9-63.9)$ & & \\
\hline Household activities & & & & & & & & & & & & & & 9.62 & $<0.001$ \\
\hline No & 1,480 & 75 & 5.2 & $(4.1-6.6)$ & 171 & 11.7 & $(10.0-13.5)$ & 341 & 22.7 & $(20.5-25.1)$ & 893 & 60.4 & $(57.7-63.1)$ & & \\
\hline Yes & 112 & 12 & 10.0 & $(5.6-17.1)$ & 28 & 24.5 & $(17.2-33.7)$ & 32 & 29.8 & $(21.6-39.4)$ & 40 & 35.8 & $(27.1-45.5)$ & & \\
\hline Money & & & & & & & & & & & & & & 5.20 & 0.001 \\
\hline No & 1,496 & 76 & 5.3 & $(4.2-6.7)$ & 183 & 12.4 & $(10.7-14.3)$ & 342 & 22.7 & $(20.5-25.1)$ & 895 & 59.6 & $(56.8-62.2)$ & & \\
\hline Yes & 96 & 11 & 10.8 & $(5.9-19.2)$ & 16 & 18.4 & $(11.2-28.7)$ & 31 & 32.4 & $(23.4-43.1)$ & 38 & 38.3 & $(28.4-49.3)$ & & \\
\hline No. ADL problems & & & & & & & & & & & & & & 4.63 & 0.003 \\
\hline$<3$ & 1,548 & 83 & 5.5 & $(4.4-6.9)$ & 187 & 12.2 & $(10.6-14.0)$ & 360 & 23.1 & $(20.9-25.4)$ & 918 & 59.2 & $(56.5-61.9)$ & & \\
\hline$\geq 3$ & 44 & 4 & 7.9 & $(2.8-20.2)$ & 12 & 28.5 & $(16.5-44.4)$ & 13 & 30.3 & $(18.0-46.2)$ & 15 & 33.4 & $(20.4-49.5)$ & & \\
\hline \multicolumn{16}{|l|}{ Functional limits due } \\
\hline to emotional health & & & & & & & & & & & & & & 63.39 & $<0.001$ \\
\hline No & 1,303 & 56 & 4.4 & $(3.4-5.7)$ & 105 & 8.4 & $(6.9-10.2)$ & 272 & 20.5 & $(18.3-22.9)$ & 870 & 66.7 & $(63.9-69.4)$ & & \\
\hline Yes & 293 & 30 & 10.1 & $(7.1-14.3)$ & 93 & 30.7 & $(25.6-36.4)$ & 102 & 35.2 & $(29.6-41.3)$ & 68 & 23.9 & $(19.1-29.4)$ & & \\
\hline \multicolumn{16}{|l|}{ Social support } \\
\hline Someone to talk to & & & & & & & & & & & & & & 5.99 & $<0.001$ \\
\hline No & 113 & 13 & 13.0 & $(7.5-21.6)$ & 8 & 6.3 & $(3.1-12.4)$ & 18 & 15.5 & $(9.7-23.7)$ & 74 & 65.2 & $(55.4-73.9)$ & & \\
\hline Yes & 1,479 & 75 & 5.1 & $(4.1-6.4)$ & 192 & 13.3 & $(11.6-15.3)$ & 353 & 23.7 & $(21.5-26.2)$ & 859 & 57.8 & $(55.0-60.5)$ & & \\
\hline $\begin{array}{l}\text { Someone to make you } \\
\text { feel cared for }\end{array}$ & & & & & & & & & & & & & & 1.30 & 0.273 \\
\hline No & 94 & 10 & 10.0 & $(5.2-18.6)$ & 14 & 14.3 & $(8.4-23.3)$ & 17 & 19.6 & $(12.1-30.1)$ & 53 & 56.1 & $(45.2-66.4)$ & & \\
\hline Yes & 1,502 & 77 & 5.3 & $(4.2-6.6)$ & 186 & 12.7 & $(11.0-14.6)$ & 356 & 23.5 & $(21.3-25.9)$ & 883 & 58.5 & $(55.8-61.3)$ & & \\
\hline
\end{tabular}

Frequencies show actual counts; percentages have been weighted.

Counts may not add up due to missing values.

$\mathrm{X}^{2}$ statistics and $p$-values are weighted outcomes from Pearson's Chi square tests with Rao \& Scott corrections.

${ }^{+}$Other category includes obsessive compulsive disorder $(n=2)$, panic disorder $(n=8)$, and mixed anxiety and depressive disorder $(n=17)$.

CMD, Common Mental Disorder; CIS-R Clinical Interview Schedule revised; ADL, activities in daily life.

\section{Discussion}

While determinants of formal help have been frequently researched, those of informal help have been underresearched. To our knowledge, this is the first time that predictors of informal help have been systematically investigated in a community survey of adults in the UK. We found that informal help is extremely commonly used among the whole sample as well as among 'cases'. Among the 'cases', $62.6 \%$ had sought informal help, whether on its own (33.6\%) or together with formal help (29\%) with $26.3 \%$ not having sought any help at all. Only $11.1 \%$ had sought formal help on its own.

We tested three hypotheses. Hypothesis 1 was that, compared to those who use formal help, exclusive use of informal help would be associated with less severe mental disorder. This was supported. Those with a less severe score on the CIS-R, not having depression, and not having suicidal ideation were more likely to exclusively seek informal help. Informal help-seekers were also more likely to rate themselves as more healthy, and less likely to report longstanding illnesses or functional limitations due to emotional health.

Contrary to our second hypothesis, we found no evidence that higher social support was associated with exclusive use of informal help seeking. We did however find that those with social support seemed less likely to seek formal help on its own. This supports Woodward et al. [13] who found that those with larger social networks were more likely to use both formal and informal help amongst their African American and black Caribbean participants.

When we tested Hypothesis 3 and compared those who had exclusively used informal help and those who 
Table 4 Logistic regression analyses comparing informal (only) help users with formal help users $(N=666)$

\begin{tabular}{|c|c|c|c|c|c|c|c|c|c|}
\hline & \multirow[b]{2}{*}{$\mathbf{N}$} & \multicolumn{2}{|c|}{ Informal only } & \multicolumn{3}{|c|}{ Unadjusted } & \multicolumn{3}{|c|}{ Adjusted $^{\ddagger}$} \\
\hline & & $\mathrm{n}$ & $\%$ & OR & $(95 \% \mathrm{Cl})$ & $p$ & $\overline{\mathrm{OR}}$ & $(95 \% \mathrm{Cl})$ & $p$ \\
\hline \multicolumn{10}{|l|}{ Socio-demographic and SES } \\
\hline Gender & & & & & & 0.991 & & & 0.958 \\
\hline Male & 231 & 130 & 55.9 & 1.0 & & & 1.0 & & \\
\hline Female & 435 & 247 & 55.8 & 1.0 & $(0.7-1.4)$ & & 1.0 & $(0.7-1.5)$ & \\
\hline Age & & & & & & 0.013 & & & 0.555 \\
\hline $16-25$ & 167 & 108 & 64.2 & 1.0 & & & 1.0 & & \\
\hline $26-40$ & 241 & 141 & 58.7 & 0.8 & $(0.5-1.2)$ & & 0.7 & $(0.4-1.2)$ & \\
\hline $41-55$ & 164 & 80 & 48.3 & 0.5 & $(0.3-0.8)$ & & 0.5 & $(0.3-0.9)$ & \\
\hline 56 or older & 94 & 48 & 51.4 & 0.6 & $(0.3-1.0)$ & & 0.9 & $(0.5-1.9)$ & \\
\hline Ethnic group & & & & & & 0.528 & & & 0.323 \\
\hline White & 422 & 231 & 54.3 & 1.0 & & & 1.0 & & \\
\hline Black Caribbean & 59 & 34 & 55.7 & 1.1 & $(0.6-1.9)$ & & 0.8 & $(0.4-1.6)$ & \\
\hline Black African & 78 & 46 & 56.1 & 1.1 & $(0.6-1.8)$ & & 0.6 & $(0.3-1.2)$ & \\
\hline Asian & 27 & 20 & 72.4 & 2.2 & $(0.9-5.5)$ & & 1.3 & $(0.4-3.9)$ & \\
\hline Other & 78 & 45 & 58.7 & 1.2 & $(0.7-2.0)$ & & 1.4 & $(0.8-2.5)$ & \\
\hline Relationship status & & & & & & 0.653 & & & 0.597 \\
\hline Married/cohabitating & 265 & 154 & 56.9 & 1.0 & & & 1.0 & & \\
\hline Non-married/cohabitating* & 401 & 223 & 55.1 & 0.9 & $(0.7-1.3)$ & & 0.9 & $(0.6-1.3)$ & \\
\hline Migration status & & & & & & 0.189 & & & 0.415 \\
\hline Non-migrant & 382 & 206 & 53.8 & 1.0 & & & 1.0 & & \\
\hline Migrant & 280 & 170 & 59.2 & 1.2 & $(0.9-1.7)$ & & 1.2 & $(0.8-1.8)$ & \\
\hline Employment & & & & & & 0.006 & & & 0.145 \\
\hline Employed & 335 & 210 & 62.0 & 1.0 & & & 1.0 & & \\
\hline Unemployed & 88 & 41 & 46.6 & 0.5 & $(0.3-0.9)$ & & 0.8 & $(0.4-1.4)$ & \\
\hline Student & 103 & 63 & 60.4 & 0.9 & $(0.6-1.5)$ & & 0.7 & $(0.4-1.3)$ & \\
\hline Other & 136 & 61 & 46.2 & 0.5 & $(0.3-0.8)$ & & 0.5 & $(0.3-0.9)$ & \\
\hline Education & & & & & & 0.022 & & & 0.910 \\
\hline No qualifications & 81 & 37 & 46.1 & 0.6 & $(0.3-1.0)$ & & 0.9 & $(0.5-1.9)$ & \\
\hline GCSE & 145 & 78 & 53.5 & 0.8 & $(0.5-1.1)$ & & 1.0 & $(0.6-1.8)$ & \\
\hline A-level & 176 & 100 & 56.6 & 0.9 & $(0.6-1.3)$ & & 1.1 & $(0.7-1.8)$ & \\
\hline Degree or above & 264 & 162 & 60.4 & 1.0 & & & 1.0 & & \\
\hline Annual household income & & & & & & $<0.001$ & & & 0.176 \\
\hline$£ 0-5,475$ & 67 & 20 & 32.0 & 0.3 & $(0.2-0.6)$ & & 0.5 & $(0.2-1.0)$ & \\
\hline$£ 5,476-12,097$ & 93 & 48 & 51.6 & 0.7 & $(0.4-1.2)$ & & 1.0 & $(0.5-1.9)$ & \\
\hline$£ 12,098-20,753$ & 79 & 49 & 60.2 & 1.0 & $(0.6-1.8)$ & & 1.3 & $(0.7-2.4)$ & \\
\hline$£ 20,754-31,494$ & 70 & 43 & 60.7 & 1.1 & $(0.6-1.9)$ & & 1.2 & $(0.6-2.1)$ & \\
\hline$£ 31,495$ or more & 261 & 156 & 59.2 & 1.0 & & & 1.0 & & \\
\hline \multicolumn{10}{|l|}{ Clinical indicators } \\
\hline CMD (CIS-R score) & & & & & & $<0.001$ & & & $<0.001$ \\
\hline No CMD $(<12)$ & 383 & 250 & 63.8 & 1.0 & & & 1.0 & & \\
\hline CMD (12-18) & 122 & 66 & 53.5 & 0.7 & $(0.4-1.0)$ & & 0.6 & $(0.4-1.0)$ & \\
\hline Symptoms likely to require treatment $(\geq 18)$ & 159 & 60 & 39.5 & 0.4 & $(0.2-0.6)$ & & 0.4 & $(0.3-0.7)$ & \\
\hline
\end{tabular}


Table 4 Logistic regression analyses comparing informal (only) help users with formal help users $(\mathrm{N}=666)($ Continued)

\begin{tabular}{|c|c|c|c|c|c|c|c|c|c|}
\hline Any CIS-R primary diagnosis & & & & & & $<0.001$ & & & 0.002 \\
\hline No & 350 & 231 & 64.0 & 1.0 & & & 1.0 & & \\
\hline Yes & 314 & 145 & 47.1 & 0.5 & $(0.4-0.7)$ & & 0.6 & $(0.4-0.8)$ & \\
\hline Non-specified neurotic disorder & & & & & & 0.223 & & & 0.252 \\
\hline No & 602 & 336 & 55.0 & 1.0 & & & 1.0 & & \\
\hline Yes & 62 & 40 & 63.8 & 1.4 & $(0.8-2.6)$ & & 1.5 & $(0.7-3.0)$ & \\
\hline Generalised anxiety disorder & & & & & & 0.175 & & & 0.344 \\
\hline No & 613 & 352 & 56.6 & 1.0 & & & 1.0 & & \\
\hline Yes & 51 & 24 & 46.3 & 0.7 & $(0.4-1.2)$ & & 0.7 & $(0.4-1.4)$ & \\
\hline Phobia (any) & & & & & & 0.847 & & & 0.855 \\
\hline No & 635 & 359 & 55.7 & 1.0 & & & 1.0 & & \\
\hline Yes & 29 & 17 & 57.6 & 1.1 & $(0.5-2.3)$ & & 1.1 & $(0.4-2.8)$ & \\
\hline Depression & & & & & & $<0.001$ & & & $<0.001$ \\
\hline No & 517 & 325 & 61.4 & 1.0 & & & 1.0 & & \\
\hline Yes & 147 & 51 & 37.1 & 0.4 & $(0.3-0.5)$ & & 0.4 & $(0.2-0.6)$ & \\
\hline Other primary CIS-R diagnosis ${ }^{\dagger}$ & & & & & & 0.952 & & & 0.895 \\
\hline No & 639 & 363 & 55.8 & 1.0 & & & 1.0 & & \\
\hline Yes & 25 & 13 & 56.4 & 1.0 & $(0.4-2.3)$ & & 0.9 & $(0.4-2.3)$ & \\
\hline Suicidal ideation & & & & & & $<0.001$ & & & $<0.001$ \\
\hline No & 604 & 363 & 59.0 & 1.0 & & & 1.0 & & \\
\hline Yes & 59 & 12 & 21.9 & 0.2 & $(0.1-0.4)$ & & 0.2 & $(0.1-0.4)$ & \\
\hline Hazardous alcohol use & & & & & & 0.856 & & & 0.484 \\
\hline No & 502 & 290 & 56.0 & 1.0 & & & 1.0 & & \\
\hline Yes & 161 & 85 & 55.2 & 1.0 & $(0.7-1.4)$ & & 1.2 & $(0.8-1.8)$ & \\
\hline Drug use (past year) & & & & & & 0.882 & & & 0.879 \\
\hline No & 481 & 273 & 55.7 & 1.0 & & & 1.0 & & \\
\hline Yes & 183 & 103 & 56.4 & 1.0 & $(0.7-1.5)$ & & 1.0 & $(0.7-1.6)$ & \\
\hline Long-standing illness & & & & & & $<0.001$ & & & 0.003 \\
\hline No & 362 & 238 & 64.9 & 1.0 & & & 1.0 & & \\
\hline Yes & 300 & 137 & 46.8 & 0.5 & $(0.3-0.7)$ & & 0.5 & $(0.4-0.8)$ & \\
\hline No. ADL problems & & & & & & 0.269 & & & 0.305 \\
\hline$<3$ & 630 & 360 & 56.5 & 1.0 & & & 1.0 & & \\
\hline$\geq 3$ & 29 & 13 & 45.5 & 0.6 & $(0.3-1.4)$ & & 0.6 & $(0.2-1.6)$ & \\
\hline Self-rated health & & & & & & $<0.001$ & & & 0.024 \\
\hline Good or better & 493 & 303 & 60.8 & 1.0 & & & 1.0 & & \\
\hline Fair or poor & 169 & 73 & 43.9 & 0.5 & $(0.4-0.7)$ & & 0.6 & $(0.4-0.9)$ & \\
\hline Functional limits due to emotional health & & & & & & $<0.001$ & & & $<0.001$ \\
\hline No & 433 & 272 & 61.5 & 1.0 & & & 1.0 & & \\
\hline Yes & 169 & 102 & 46.3 & 0.5 & $(0.4-0.8)$ & & 0.5 & $(0.3-0.8)$ & \\
\hline \multicolumn{10}{|l|}{ Social support } \\
\hline Someone to talk to & & & & & & 0.174 & & & 0.456 \\
\hline No & 433 & 18 & 44.5 & 1.0 & & & 1.0 & & \\
\hline Yes & 225 & 353 & 56.3 & 1.6 & $(0.8-3.2)$ & & 1.4 & $(0.6-3.5)$ & \\
\hline
\end{tabular}


Table 4 Logistic regression analyses comparing informal (only) help users with formal help users $(\mathrm{N}=666)($ Continued)

\begin{tabular}{llllllll}
\hline $\begin{array}{l}\text { Someone to make you feel cared for } \\
\text { No }\end{array}$ & & & & & 0.172 & 0.521 \\
Yes & 69 & 17 & 44.6 & 1.0 & & 1.0 \\
\hline
\end{tabular}

Frequencies show actual counts, percentages and regression analyses have been weighted.

Counts may not add up due to missing values.

p-values for age, education, income and CMD test for trends.

*Non-married/cohabitating category include single, divorced/separated, and widowed relationship status.

${ }^{\dagger}$ Other category includes obsessive compulsive disorder $(n=2)$, panic disorder $(n=7)$, and mixed anxiety and depressive disorder $(n=16)$.

${ }^{\ddagger}$ Socio-demographic and socio-economic indicators adjustfor each other, without including clinical indicators or social support variables in the model. Clinical indicators and social support variables are tested in separate models adjusting for all socio-demographic and SES indicators. These models use adjust for age using the continuous measure.

SES, socio-economic status; CMD, Common Mental Disorder; CIS-R Clinical Interview Schedule revised; ADL, activities in daily life.

had sought formal help, we found no differences in sociodemographic factors (age, gender or ethnic group), when unadjusted and adjusted for clinical factors. With Hypothesis 4 , in the unadjusted model, those in lower SES groups were found to be less likely to use informal help but this association was rendered non-significant when models were adjusted for clinical severity. These results suggest that it is not socio-demographic or socio-economic factors that drive informal or formal help-seeking, but clinical factors such as severity and complexity that do.

This study has some limitations. The survey took place in an urban area in London so that findings from this study may not be generalisable to all communities. This is an area with greater access to more services, because of the presence of a very large mental health trust. However, there is also likely to be a higher level of need and mental health difficulties because of the higher deprivation level of the area. A national survey showed that in London, there are slightly higher rates of stigma, as indicated by intended contact with a person with a mental illness [33] but that a greater reduction in stigma also occurred over time [34]. Given this, it is likely that these results would be generalizable to other urban areas but less so to rural areas. The ethnic population is also different from that in other areas, comprising more black Caribbeans and black Africans and fewer Asians. And while attempts were made to create adequate groupings, some cells (e.g. ethnic minority groups) were small. In terms of service use assessment, self-report may be open to recall bias; however, other studies have largely shown that self-reported service use shows reasonable agreement with other sources, including administrative records among those with measured mental health need [35]. The cross-sectional nature of the survey also limits our ability to understand the causal relationship between different types of help-seeking and the longitudinal patterns of access to help. The wording of the question may have affected responses as formal help related to 'anxious or depressed or a mental, nervous or emotional problem' whereas informal help related to 'an emotional problem'.
The 4 help-seeking group categorization used in this study is similar to that previously used by Woodward and colleagues [13] in their study of African Americans and Black Caribbeans with lifetime mood, anxiety or substance misuse problems. They found 23\% had used informal help only, $41 \%$ had used both informal and formal help, $14 \%$ used formal help only and $22 \%$ had sought no help. These figures are slightly different from our study in which fewer 'cases' sought either informal help only or no help at all. It is likely that the differences can be explained by the greater chronicity of problems and ethnic differences of their participants. In the UK, Rudell and colleagues [14] also found a pluralistic pattern of help-seeking with GP consultation occurring alongside informal help-seeking.

Work with adolescents [12] and ethnic minorities [13], indicates seeking informal help is often preferred when seeking help for mental disorders. Our study supported those findings; the more frequent users of informal help in this study were younger people and those from ethnic minority groups. In addition, we found employed people frequently used informal help.

We also found that different help-seeking patterns varied by ethnic group. Black Caribbeans used their friends more than family members and used formal help quite frequently. Asians were more likely to use their family, but not use formal help. Black Africans were more likely to seek help from a religious leader and be less likely to seek formal help. Rudell and colleagues [14] also found ethnic differences amongst Bangladeshi and black Caribbean and white British in the choice of help-seeking strategies. They found that both the Bangladeshi and Caribbean groups used spiritual forms of help more frequently. However, Bangladeshi participants used medical help more than the other 2 groups, but informal help less frequently.

In terms of future research, there is a potentially a large research agenda. A key question is from whom people seek informal help. From this study and that by Rudell [14] there are ethnic differences which merit further research. Following on from this, barriers and facilitators to seeking informal help need investigation. Possible factors 
Table 5 The socio-demographic distribution of sources of help among informal help users $(n=577)$

\begin{tabular}{|c|c|c|c|c|c|c|c|c|c|c|c|c|c|c|c|c|c|}
\hline & \multirow[b]{2}{*}{$\mathbf{N}$} & \multicolumn{4}{|c|}{ Friend } & \multicolumn{4}{|c|}{ Family } & \multicolumn{4}{|c|}{ Religious leader } & \multicolumn{4}{|c|}{ Other } \\
\hline & & $\mathrm{n}$ & $\%$ & $(95 \% \mathrm{Cl})$ & $p$ & n & $\%$ & $(95 \% \mathrm{Cl})$ & $p$ & $\mathrm{n}$ & $\%$ & $(95 \% \mathrm{Cl})$ & $p$ & $\mathrm{n}$ & $\%$ & $(95 \% \mathrm{Cl})$ & $p$ \\
\hline Gender & & & & & 0.595 & & & & 0.562 & & & & 0.584 & & & & 0.001 \\
\hline Male & 194 & 130 & 65.3 & $(57.8-72.1)$ & & 121 & 63.0 & $(55.8-69.7)$ & & 9 & 4.4 & $(2.3-8.4)$ & & 24 & 12.6 & $(8.5-18.3)$ & \\
\hline Female & 383 & 267 & 67.6 & $(62.4-72.4)$ & & 249 & 65.5 & $(60.5-70.2)$ & & 18 & 5.5 & $(3.4-8.6)$ & & 18 & 4.7 & $(2.9-7.4)$ & \\
\hline Age & & & & & $<0.001$ & & & & 0.526 & & & & 0.047 & & & & 0.736 \\
\hline $16-25$ & 148 & & 120 & 81.9 & $(74.7-87.4)$ & & 95 & 63.1 & $(54.0-71.2)$ & & 3 & 2.1 & $(0.7-6.4)$ & & 7 & 4.8 & $(2.3-9.8)$ \\
\hline $26-40$ & 220 & 153 & 70.2 & $(63.8-75.9)$ & & 136 & 62.0 & $(55.7-67.9)$ & & 8 & 3.6 & $(1.7-7.7)$ & & 17 & 6.6 & $(4.1-10.4)$ & \\
\hline $41-55$ & 134 & 86 & 62.0 & (53.1-70.1) & & 86 & 66.2 & (57.5-73.8) & & 9 & 6.6 & $(3.3-12.5)$ & & 12 & 8.2 & $(4.6-14.1)$ & \\
\hline 56 or older & 75 & 38 & 50.6 & (38.9-62.3) & & 53 & 70.5 & $(58.8-80.0)$ & & 7 & 10.0 & $(4.8-19.6)$ & & 6 & 7.0 & $(3.1-15.1)$ & \\
\hline Ethnic group & & & & & 0.004 & & & & 0.040 & & & & $<0.001$ & & & & 0.238 \\
\hline White & 365 & 254 & 67.4 & $(61.7-72.6)$ & & 237 & 65.8 & $(60.4-70.8)$ & & 10 & 2.7 & $(1.4-5.2)$ & & 32 & 8.0 & $(5.6-11.2)$ & \\
\hline Black Caribbean & 48 & 37 & 77.9 & $(63.5-87.7)$ & & 26 & 53.6 & $(40.3-66.4)$ & & 2 & 3.5 & $(0.5-20.9)$ & & 3 & 4.3 & $(1.3-12.7)$ & \\
\hline Black African & 66 & 44 & 66.4 & $(53.5-77.2)$ & & 43 & 66.3 & (52.8-77.6) & & 10 & 17.8 & $(9.4-31.3)$ & & 2 & 2.8 & $(0.7-10.7)$ & \\
\hline Asian & 26 & 9 & 32.6 & $(17.4-52.7)$ & & 22 & 88.4 & $(68.5-96.4)$ & & 3 & 14.9 & $(4.9-37.4)$ & & 3 & 11.1 & $(3.4-30.4)$ & \\
\hline Other & 70 & 52 & 72.0 & $(58.9-82.1)$ & & 41 & 58.3 & $(46.2-69.5)$ & & 2 & 5.1 & $(1.3-18.1)$ & & 2 & 3.1 & $(0.8-11.6)$ & \\
\hline Migration status & & & & & 0.662 & & & & 0.882 & & & & 0.004 & & & & 0.203 \\
\hline Non-migrant & 326 & 227 & 67.9 & $(62.0-73.3)$ & & 211 & 65.2 & $(59.6-70.5)$ & & 9 & 2.6 & $(1.2-5.4)$ & & 27 & 7.7 & $(5.2-11.1)$ & \\
\hline Migrant & 250 & 170 & 66.0 & $(59.5-72.0)$ & & 159 & 64.6 & $(58.3-70.5)$ & & 18 & 8.7 & $(5.3-13.9)$ & & 14 & 5.0 & $(2.9-8.5)$ & \\
\hline
\end{tabular}

Frequencies show actual counts; percentages have been weighted.

Counts may not add up due to missing values.

$\mathrm{p}$-values show significance level of Pearson's Chi square test with Rao \& Scott corrections. 
may be gender, ethnic background, characteristics of social networks and emotional competence. In our study, men, older adults, those with low educational qualifications and low income were less likely to use informal help. Closer examination of the attitudes of these groups to informal help would be useful.

Pathways from informal help to formal help are extremely important particularly for severe problems. Where formal help is indicated, it has been suggested that informal help could act as a bridge to access help for mental disorders [36] or as an early intervention because formal resources are not always available [37]. A key question is what determines the decision to seek formal help. Possible triggers may be family support [13] and severity of problems [13]. However, there is also evidence that transferring from informal to formal help may not always occur. Lamb [17] found that barriers for 'hard to reach' groups, included withdrawal from wider social networks in order to protect their core identities in these communities, fearing difficulties would be labeled and stigmatized. These groups experienced the interface with primary care being difficult because of differences in values. There is also some anecdotal evidence that religious leaders offering informal support to people attending their church, may not always assist in the transition to formal help, often continuing to provide support themselves (Codjoe, personal communication).

The effectiveness of informal help is a crucial question. What constitutes informal help might be difficult to define, as by their nature, this help varies, and is not often sought regularly or consistently. It is by definition much more difficult to assess informal help because researchers are rarely present when the person with the mental health problem first approaches a friend or a member of the family. Less conventional designs may need to be used. Studies might need to be retrospective or if prospective, focus around a life problem (e.g. unemployment, traumatic incident) or with selected groups where longitudinal follow-ups are possible (e.g. college students). However, Pfeiffer et al. [38] conducted a metaanalysis of peer support interventions and found these were more effective than care as usual, and as effective as group CBT for depression. Additionally, individual characteristics of informal helpers and those helped merit investigation. One area may be the components of skilful informal help-giving. In a qualitative study, Griffiths et al. [39] found that the informal help can have many advantages such as social, emotional, informational and companionship support but there were also some disadvantages namely, stigma and inappropriate support. It would be also important to investigate characteristics of people who are easier and more difficult to help.

Finally, we found $26.3 \%$ of 'cases' did not seek any kind of help. This is consistent with findings by Oliver et al. who found that over $20 \%$ with more severe mental health problems were non-help-seekers. It is important to recognize that not all people with mental health problems will need formal help from services. Sareen et al. [40] found that $50 \%$ with diagnosable problems remitted without intervention at 3 year follow-up. Whiteford et al. [41] who examined untreated samples such as waiting list participants, estimated that over 53\% with diagnosed depression would remit after 12 months and found that severity affected remission. Information about the use of informal help was not available in either study. It would be helpful to conduct a closer investigation of those who spontaneously remit to see if informal help has been relevant, and to whom.

\section{Service implications}

Given how frequently informal help is used, much more attention should be paid to informal networks. Working with patients' social networks could lead to more accessible and possibly better outcomes. Planning services around social networks of different ethnic and social groups could help better target services. Thus, offering help to families of Asian and older people with mental health problems could match the patterns for these groups whereas offering help to friends of black Caribbeans would be more natural for that group. It may be that once we better understand the role of informal and formal help for people in a population, then we would be in a better position to implement Kleinman's [7] ideas about matching perceptions of problems, and then offering interventions that 'fit' better. Access for 'hard to engage' groups could include both raising awareness of services in primary care for these groups, as well as services bridging the 'gap' through understanding these people's positions and perceptions better, resulting in a broader 'treatment' [17]. Involving primary care services in identifying these 'hard to engage' people has been attempted, although the numbers were small and the success limited [42]. A key issue about the use of informal help is how professionals feel about it as they do not always endorse this $[4,5]$. Nevertheless, informal help relates to the optimal mix of services proposed by the World Health Organisation (WHO) with self-care and informal community care being the bottom 2 layers prior to primary care services [6]. They propose that these informal services can be both helpful in preventing demands being made in the formal services, as well as be helpful when people are discharged from the formal services. While proposed with less developed countries in mind, this could be a useful model globally.

\section{Conclusions}

This study has shown that informal help-seeking is an extremely important and commonly used process. Among 
the 'cases', $62.6 \%$ had sought informal help, whether on its own $(33.6 \%)$ or together with formal help (29\%) with $26.3 \%$ not having sought any help at all. Only $11.1 \%$ had sought formal help on its own. Many papers focus on the statistic that only a third of people with mental health problems seek formal help. To this statistic needs to be added another - that a third seek informal help only, which leaves only a third not seeking any help at all. If we are to harness informal help, then we urgently need to research its use, as well as its effectiveness.

\section{Competing interests}

The authors declare that they have no competing interests.

\section{Authors' contributions}

$J B$ and $\mathrm{MH}$ designed the study and LA analysed the data. JB drafted the paper and all the other authors $(\mathrm{MH}, \mathrm{SEL}, \mathrm{LA}, \mathrm{MJH}, \mathrm{SH})$ read and revised it critically for important intellectual content. All the authors have given final approval of the version to published. All the authors agree to be accountable for all aspects of the work in ensuring that questions related to the accuracy or integrity of any part of the work are appropriately investigated and resolved and to jointly take public responsibility for the content of the paper.

\section{Authors' information}

All authors are employed by King's College London.

JSLB (PhD, M. Psychol (clin), BSc) is a Senior Lecturer in Clinical Psychology in the Department of Psychology.

SEL (MHS, PhD) is a Lecturer in the Health Service and Population Research Department.

LA (BSC MSc) is a PhD Student in the Department of Psychological Medicine. MJH (MBBS MSC PhD MRCP MRCPsych) is a Senior Lecturer in Epidemiological \& Occupational Psychiatry in the Department of Psychological Medicine. SLH (BA MA PhD) is a Senior Lecturer in the Department of Psychological Medicine.

$\mathrm{MH}$ (PhD, MRCPsych) is a Professor of General Hospital Psychiatry in the Department of Psychological Medicine.

\section{Acknowledgements}

This research was supported by the Biomedical Research Nucleus data management and informatics facility at South London and Maudsley NHS Foundation Trust, which is funded by the National Institute for Health Research (NIHR) Mental Health Biomedical Research Centre at South London and Maudsley NHS Foundation Trust and King's College London and a joint infrastructure grant from Guy's and St Thomas' Charity and the Maudsley Charity.

SL Hatch, M Hotopf and M Henderson receive salary support from the National Institute for Health Research (NIHR) Mental Health Biomedical Research Centre at South London and Maudsley NHS Foundation Trust and King's College London. L Aschan receives an Institute of Psychiatry Excellence Studentship from the Institute of Psychiatry, King's College London. SEL has received consulting fees from Lundbeck.

The views expressed are those of the authors and not necessarily those of the NHS, the NIHR or the Department of Health. The funders did not have a role in the study design; collection, analysis or interpretation of data; the writing of the manuscript; or in the decision to submit the manuscript for publication

\section{Author details}

${ }^{1}$ Psychology Department (PO77), Kings College London, Institute of Psychiatry, De Crespigny Park, London SE5 8AF, UK. ${ }^{2}$ Health Services and Population Research Department, (PO29), Institute of Psychiatry, De Crespigny Park, London SE5 8AF, UK. '3Department of Psychological Medicine, Weston Education Centre, Institute of Psychiatry, Cutcombe Road, London SE5 9RJ, UK.

Received: 18 May 2014 Accepted: 20 September 2014

Published online: 08 October 2014
References

1. Andrews G, Sanderson $K$, Slade T, Issakidis C: Why does the burden of disease persist? Relating the burden of anxiety and depression to effectiveness of treatment. Bull World Health Organ 2000, 78(4):446-454

2. Kessler RC, Chiu WT, Demler O, Merikangas KR, Walters EE: Prevalence, severity, and comorbidity of 12-month DSM-IV disorders in the National Comorbidity Survey Replication. Arch Gen Psychiatry 2005, 62(6):617-627.

3. Alonso J, Angermeyer MC, Bernert S, Bruffaerts R, Brugha T, Bryson H, Girolamo G, Graaf R, Demyttenaere K, Gasquet I: Use of mental health services in Europe: results from the European Study of the Epidemiology of Mental Disorders (ESEMeD) project. Acta Psychiatr Scand 2004, 109(s420):47-54

4. Jorm AF, Korten AE, Jacomb PA, Rodgers B, Pollitt P: Beliefs about the helpfulness of interventions for mental disorders: a comparison of general practitioners, psychiatrists and clinical psychologists. Aust N Z 」 Psychiatry 1997, 31(6):844-851.

5. Morgan AJ, Reavley NJ, Jorm AF: Beliefs about mental disorder treatment and prognosis: comparison of health professionals with the Australian public. Aust N Z J Psychiatry 2014, 48(5):442-451.

6. World Health Organisation: Improving Health Systems and Services for Mental Health. [http://whqlibdoc.who.int/publications/2009/9789241 598774_eng.pdf?ua=1]

7. Kleinman A, Eisenberg L, Good B: Culture, illness, and care: clinical lessons from anthropologic and cross-cultural research. Ann Intern Med 1978, 88(2):251-258.

8. Kirmayer $\sqcup$ : Cultural variations in the response to psychiatric disorders and emotional distress. Soc Sci Med 1989, 29(3):327-339.

9. Campinha-Bacote J: The process of cultural competence in the delivery of healthcare services: a model of care. J Transcult Nurs 2002, 13(3):181-184.

10. Hays RB, Catania JA, Mckusick L, Coates TJ: Help-seeking for AIDS-related concerns: a comparison of gay men with various HIV diagnoses. Am J Community Psychol 1990, 18(5):743-755.

11. Ansara DL, Hindin MJ: Formal and informal help-seeking associated with women's and men's experience of intimate partner violence in Canada. Soc Sci Med 2010, 70:1011-1018.

12. Rickwood D, Deane FP, Wilson CJ, Ciarrochi J: Young people's help-seeking for mental health problems. Adv Ment Health 2005, 4(3):218-251.

13. Woodward AT, Taylor RJ, Bullard KM, Neighbors HW, Chatters LM, Jackson JS: Use of professional and informal support by African Americans and Caribbean blacks with mental disorders. Psychiatr Serv 2008, 59(11):1292-1298.

14. Rudell K, Bhui K, Priebe S: Do 'alternative' help-seeking strategies affect primary care service use? A survey of help-seeking for mental distress. BMC Public Health 2008, 8:207.

15. Oliver Ml, Pearson N, Coe N, Gunnell D: Help-seeking behaviour in men and women with common mental health problems: cross-sectional study. Br J Psychiatry 2005, 186:297-301

16. Goldberg D, Williams P: A User's Guide to the General Health Questionnaire. Windsor, Berks: NFER-Nelson; 1988.

17. Lamb J, Bower P, Rogers A, Dowrick C, Gask L: Access to mental health in primary care: a qualitative meta-synthesis of evidence from the experience of people from 'hard to reach' groups. Health 2012, 16(1):76-104.

18. Bebbington PE, Meltzer $H$, Brugha TS, Farrell M, Jenkins R, Ceresa C, Lewis G: Unequal access and unmet need: neurotic disorders and the use of primary care services. Psychol Med 2000, 30(6):1359-1367.

19. Mojtabai R, Olfson M, Sampson NA, Jin R, Druss B, Wang PS, Wells KB, Pincus HA, Kessler RC: Barriers to mental health treatment: results from the National Comorbidity Survey Replication. Psychol Med 2011, 41(8):1751-1761.

20. Shaw CM, Creed F, Tomenson B, Riste L, Cruickshank JK: Prevalence of anxiety and depressive illness and help seeking behaviour in African Caribbeans and white Europeans: two phase general population survey. BMJ 1999, 318(7179):302-305.

21. McManus S, Meltzer H, Brugha T, Bebbington P, Jenkins R: Adult Psychiatric Morbidity in England 2007: Results of a Household Survey. 2009 [http://www.hscic.gov.uk/pubs/psychiatricmorbidity07]

22. Hatch SL, Frissa S, Verdecchia M, Stewart R, Fear NT, Reichenberg A, Morgan C, Kankulu B, Clark J, Gazard B, Medcalf R, SELCoH study team, Hotopf M: Identifying socio-demographic and socioeconomic determinants of health inequalities in a diverse London community: the South East London Community Health (SELCoH) study. BMC Public Health 2011 11(1):861. 
23. Hatch SL, Woodhead C, Frissa S, Fear NT, Verdecchia M, Stewart R, Reichenberg A, Morgan C, Bebbington P, McManus S, Brugha T, Kankulu B, Clark JL, Gazard B, Medcalf R, Hotopf M, SELCoH Study Team: Importance of thinking locally for mental health: data from cross-sectional surveys representing South East London and England. PLOS ONE 2012, 7(12):e48012.

24. Office of National Statistics: NOMIS Labour Market Profile: Southwark. [https://www.nomisweb.co.uk/reports/Imp/ward/1396703432/report.aspx]

25. Office of National Statistics: NOMIS Labour Market Profile: Lambeth. [https:/www.nomisweb.co.uk/reports/lmp/ward/1396703429/report.aspx]

26. Department of Health: Health Profile 2011: Southwark. [http://www.apho. org.uk/resource/item.aspx?RID=105412]

27. Department of Health: Health Profile 2011: Lambeth. [http://www.apho. org.uk/resource/item.aspx?RID=105406]

28. Lewis G: Assessing psychiatric disorder with a human interviewer or a computer. J Epidemiol Community Health 1994, 48(2):207-210.

29. Ware JE Jr, Kosinski M, Keller SD: A 12-Item Short-Form Health Survey: construction of scales and preliminary tests of reliability and validity. Med Care 1996, 34(3):220-233.

30. Babor T, Higgins-Biddle J, Saunders J, Monteiro M: The Alcohol Use Disorders Identification Test: Guidelines for Use in Primary Care. 2nd edition. Geneva: World Health Organisation; 2001.

31. Pickles A, Dunn G, Vazquez-Barquero JL: Screening for stratification in twophase ('two-stage') epidemiological surveys. Stat Methods Med Res 1995, 4:73-89.

32. Archer K, Lemeshow S: Goodness-of-fit test for a logistic regression model fitted using survey sample data. Stata J 2006, 6(1):97-105.

33. Evans-Lacko S, Malcolm E, West K, Rose D, London J, Rusch N, Little K, Henderson C, Thornicroft G: Influence of time to change's social marketing interventions on stigma in England 2009-2011. Br J Psychiatry 2013, 202(s55):s77-s88.

34. Evans-Lacko S, Corker E, Williams P, Henderson C, Thornicroft G: Trends in mental illness related public stigma among the English population in 2003-2013: influence of the Time to Change anti-stigma campaign. Lancet Psychiatry in press.

35. Palin JL, Goldner EM, Koehoorn M, Hertzman C: Prevalence and frequency of mental health care provided by general practitioners: differences between 2 national data sources for the same population. Can J Psychiatry 2012, 57(6):366-374.

36. Stanton J, Randal P: Doctors accessing mental-health services: an exploratory study. BMJ Open 2011, 1(1):e000017.

37. Jorm AF: Mental health literacy: empowering the community to take action for better mental health. Am Psychol 2012, 67(3):231-243.

38. Pfeiffer PN, Heisler M, Piette JD, Rogers MA, Valenstein M: Efficacy of peer support interventions for depression: a meta-analysis. Gen Hosp Psychiatry 2011, 33(1):29-36

39. Griffiths KM, Crisp DA, Barney L, Reid R: Seeking help for depression from family and friends: a qualitative analysis of perceived advantages and disadvantages. BMC Psychiatr 2011, 11:196.

40. Sareen J, Henriksen CA, Stein MB, Afifi TO, Lix LM, Enns MW: Common mental disorder diagnosis and need for treatment are not the same: findings from a population-based longitudinal survey. Psychol Med 2013, 43(9):1941-1951.

41. Whiteford HA, Harris MG, McKeon G, Baxter A, Pennell C, Barendregt JJ, Wang J: Estimating remission from untreated major depression: a systematic review and meta-analysis. Psychol Med 2013, 43(8):1569-1585.

42. Dowrick C, Chew-Graham C, Lovell K, Lamb J, Aseem S, Beatty S, Bower P, Burroughs H, Clarke P, Edwards S, Gabbay M, Gravenhorst K, Hammond J, Hibbert D, Kovandžić M, Lloyd-Williams M, Waheed W, Gask L: Increasing equity of access to high-quality mental health services in primary care: a mixed-methods study. Programme Grants Appl Res 2013, 1(2).

doi:10.1186/s12888-014-0275-y

Cite this article as: Brown et al: Seeking informal and formal help for mental health problems in the community: a secondary analysis from a psychiatric morbidity survey in South London. BMC Psychiatry 2014 14:275

\section{Submit your next manuscript to BioMed Central and take full advantage of:}

- Convenient online submission

- Thorough peer review

- No space constraints or color figure charges

- Immediate publication on acceptance

- Inclusion in PubMed, CAS, Scopus and Google Scholar

- Research which is freely available for redistribution

Submit your manuscript at www.biomedcentral.com/submit
Biomed Central 